\title{
Correction to: Vulnerability of coffee (Coffea spp.) genetic resources in the United States
}

\author{
Sarada Krishnan (1) Tracie Matsumoto - Chifumi Nagai - James Falconer • \\ Suzanne Shriner $\cdot$ Jennifer Long $\cdot$ Juan F. Medrano $\cdot$ Fernando E. Vega $(\mathbb{D}$
}

Published online: 12 June 2021

(C) Springer Nature B.V. 2021

Correction to: Genet Resour Crop Evol

https://doi.org/10.1007/s10722-021-01217-1

Unfortunately, the article title is wrongly published in the original publication of the article.

The original article can be found online at

https://doi.org/10.1007/s10722-021-01217-1.

\section{S. Krishnan $(\bowtie)$}

Horticulture and Center for Global Initiatives, Denver

Botanic Gardens, 909 York Street, Denver,

CO 80206, USA

e-mail: saradakrishnan@msn.com

\section{T. Matsumoto}

Tropical Plant Genetic Resources and Disease Research, Pacific Basin Agricultural Research Center, U.S.

Department of Agriculture, Agricultural Research

Service, 64 Nowelo Street, Hilo, HI 96720, USA

e-mail: tracie.matsumoto@usda.gov

\section{Nagai}

Hawaii Agriculture Research Center,

P. O. Box 100, Kunia, HI 96759, USA

e-mail: cnagai@harc-hspa.com

\section{J. Falconer}

MauiGrown Coffee, Inc., 277 Lahainaluna Rd., Lahaina,

HI 96761, USA

e-mail: jkimof@msn.com
The correct article title is provided here, Vulnerability of coffee (Coffea spp.) genetic resources in the United States.

The original article has been corrected.

Publisher's Note Springer Nature remains neutral with regard to jurisdictional claims in published maps and institutional affiliations.

\author{
S. Shriner \\ Synergistic Hawaii Agriculture Council, 190 Keawe \\ Street Suite 25, Hilo, HI 96720, USA \\ e-mail: suzanne@shachawaii.org \\ J. Long \\ World Coffee Research, 10940 SW Barnes Rd. \#334, \\ Portland, OR 97225, USA \\ e-mail: vern@worldcoffeeresearch.org \\ J. F. Medrano \\ University of California, Davis, CA 95616, USA \\ e-mail: jfmedrano@ucdavis.edu \\ J. F. Medrano \\ Frinj Coffee, 1362 Farren Rd, Goleta, \\ CA 93117, USA \\ F. E. Vega $(\bowtie)$ \\ Sustainable Perennial Crops Laboratory, U.S. Department \\ of Agriculture, Agricultural Research Service, Beltsville, \\ MD 20705, USA \\ e-mail: Fernando.Vega@usda.gov
}

